\title{
Interaction effect among the disease managements, seed treatments and locations in 0-9897 variety on disease incidence, seed yield, stick yield and fiber yield following line sowing method in the field
}

\begin{abstract}
The experiments were conducted in the field of Jute Agriculture Experimental Station (JAES), Manikgonj and Kishoregonj Regional Station (KRS), Kishoregonj of BJRI. The experiments were conducted during the period April 2012 to January 2013. Two different disease management viz. spraying of BAU- Biofungicide and spraying of Dithane M-45, two different seed treatments viz. Provax-200 and BAU- Biofungicide and two locations viz. JAES and KRS were used for the present study. Interaction between disease managements and seed treatments, in case of KRS, after spraying, interaction effect of BAU- Biofungicide spraying plants and BAU- Biofungicide treated seed resulted lower seed borne infection $(1.39 \%)$ and highest disease incidence $(3.46 \%)$ was encountered in interaction of control and control treatment. The fiber yield/ha (3.97 ton), stick yield/ha (8.82 ton) and seed yield/ ha $(373.54 \mathrm{~kg})$ were recorded in interaction effect of BAU- Biofungicide spraying plants and BAU- Biofungicide treated seed. In case of JAES, after spraying, lower seed borne infection $(2.53 \%)$ was observed in interaction effect of BAU- Biofungicide spraying plants and BAU- Biofungicide treated seed and highest disease incidence $(5.08 \%)$ was encountered in interaction effect among control condition and control treatment. The highest fibre yield/ha (5.03 ton), stick yield/ha (10.01 ton) and seed yield/ ha (369.02kg) were recorded in interaction effect of BAU- Biofungicide spraying plants and BAUBiofungicide treated seed. Interaction between disease managements, seed treatments and locations, after spraying, interaction effect of KRS, BAU- Biofungicide spraying plants and BAU- Biofungicide treated seed resulted lower seed borne infection $(1.36 \%)$ and highest disease incidence $(5.08 \%$ ) was encountered in interaction effect of JAES, control condition and control treatment. The highest fibre yield/ha (5.03 ton) and stick yield/ha (10.01 ton) were recorded in interaction effect of JAES, BAU- Biofungicide spraying plants and BAUBiofungicide treated seed. Highest seed yield $/ \mathrm{ha}(373.54 \mathrm{~kg})$ were recorded in interaction effect of KRS, BAU- Biofungicide spraying plants and BAU- Biofungicide treated seed.
\end{abstract}

Keywords: interaction, disease managements, seed treatments, locations, disease incidence, seed quality, yield, O-9897
Volume 4 Issue 2 - 2017

\author{
S M A Haque \\ Department of Plant Pathology, Senior Scientific Officer, \\ Bangladesh
}

\author{
Correspondence: SMA Haque, Department of Plant \\ Pathology, Senior Scientific Officer, Bangladesh Pest Management \\ Division, Jute Research Institute, Dhaka-I207, Bangladesh, \\ Email upolbjri@gmail.com
}

Received: February 14, 2017 | Published: March 01, 2017

\section{Introduction}

Jute is one of the major cash crops of Bangladesh. Its influence on ecology and economy is so intimate that it's the effects are significantly related to the agro-ecology and the socio-economic life of the people. Jute crop is also cultivated in different countries. The jute crop also greatly improves the soil fertility status by incorporating organic matter to the soil through decomposition of shaded leaves and plant residues and helps in breaking plough-pans through its long taproots. Also, jute and jute goods have been recognized as being friendly to the environment. Jute is mostly grown in the IndoBangladesh region and in some countries of Southeast Asia. Among the jute growing countries of the world, Bangladesh was second position in respect of production. ${ }^{1}$ The land and climatic conditions of Bangladesh are congenial for the production of high quality jute. In Bangladesh, about 0.709 million hectares of land was under jute cultivation and the total yield was 8.40 million bales. ${ }^{2,3}$ As per Khandakar, ${ }^{4}$ Bangladesh annually needs about 4000 metric tons of jute seeds of which only $12-15 \%$ is produced and supplied by the Bangladesh Agricultural Development Corporation (BADC). The rest of the seeds, about $85 \%$ or more of the requirement, are produced and managed by farmers'. ${ }^{5}$ Jute suffers from more than 13 different diseases Fakir $\mathrm{GA}^{6}$ and 10 of them are seed borne. Sowing of infected seeds may cause the death of seedlings and often plants escaping early infection succumb to death due to different diseases. Seed germination decreases with the increase of the seed borne infection. Seeds having higher seed borne infection results to significantly higher amount of disease development in the field. The rate of transmission of these pathogens from infected seeds to the growing plants and finally to the harvested seeds was relatively low. ${ }^{7}$ Among the seed-borne fungal diseases, stem-rot, black-band, and anthracnose caused by Macrophomina phaseolina (Tassi, Goid.), Botryodiplodia theobromae and Colletotrichum corchori ${ }^{8}$ respectively are frequently transmitted through jute seeds. ${ }^{9-11}$ Macrophomina phaseolina alone can cause $10 \%$ yield loss. ${ }^{12}$ Stem rot, black band, anthracnose, foot rot and wilt (Rhizoctonia solani) and leaf mosaic (virus) are responsible for seed rot, pre and post emergence damping off seedlings and spreads of the diseases to standing crops and loss and deterioration of quality of fibre. ${ }^{9,12-14}$ Soft rot, foot rot and wilt caused by Sclerotium rolfsii and Rhizoctonia solani, respectively also cause considerable yield losses to the crop. Cercospora leaf spot and target spot caused by Cercospora chorchori and Corynespora 
cassicola, respectively, are not so important, though these two pathogens are frequently transmitted through jute seeds. The pathogens like Fusarium spp. (Fusarium semitectum and Fusarium oxysporum), Curvularia lunataand Phomopsis sp. are responsible for causing germination failure and seed rot. ${ }^{11}$ Yield loss due to seed borne diseases of jute is $8-20 \%$ depending on the severity of jute diseases from year to year. ${ }^{15}$ Infected jute seed fail to germinate or the young seedlings emerging from the infected seed die. Infection of jute seed causes germination failure, post emergence damping off and seedling blight. ${ }^{16}$ Jute seedlings or growing plants produced in the field from the infected seeds and escaping early infection may often be infected at the later stages of their growth by the primary seed borne inocula grown and multiplied on the infected dead seeds and seedlings. Later on, these inocula may be transmitted to the healthy growing plants of the same or neighboring plants or even neighboring fields resulting to disease outbreak, often in epidemic form. Seed borne pathogens causing diseases on the growing jute plants in the field quite often attack the capsules or pods and subsequently infect the seed, resulting to production of infected or unhealthy seeds. Considering the above facts, the present study was carried out with the objective was to find out suitable disease management and seed treatments for quality jute seeds and fibre production.

\section{Materials and methods}

\section{Experimental sites and period}

The experiments were conducted in the field of Jute Agriculture Experimental Station (JAES), Manikgonj and Kishoregonj Regional Station (KRS), Kishoregonj of BJRI. The experiments were conducted during the period April 2012 to January 2013.

\section{Varieties used}

Seed of O-9897 was selected for this study.

\section{Disease management in the field}

i. Spraying of fungicide Dithane M-45 (0.2\%)

ii. Spraying of BAU-Biofungicide @, 2\% in water ${ }^{17}$

iii. Control (without spray)

\section{Spraying of Dithane M-45}

Dithane M-45 (Mancozeb 80\%) was sprayed @ 2gm/L (0.2\%) water as a preventive measure against the diseases of jute plants. Altogether, two sprays were done. The first spraying was done after disease occurred and the second spraying was done after 45 days of 1 st spraying.

\section{Spraying of BAU- Biofungicide}

BAU- Biofungicide was sprayed @ 2\% in water as a preventive measure against the diseases of jute plants. Altogether, two sprays were done. The first spraying was done after disease occurred and the second spraying was done after 45 days of $1^{\text {st }}$ spraying.

\section{Seed management}

i. Seed treated with Provax-200 WP (0.4\% of seed weight)

ii. Seed treated with BAU- Bio fungicide @ 3\% of seed weight ${ }^{17}$

iii. Control (Untreated)

\section{Seed treated with Provax -200 WP}

Seeds were treated with Provax-200 (5,6- dihydro -2- methyl-1, 4-oxathin-3- carboxinilide, Group: Oxathin) @ 0.4\% of seed weight in a $250 \mathrm{ml}$ Erlenmeyer flask and shaken thoroughly for proper coating of the seeds with the fungicides. ${ }^{6}$

\section{Seed treated with BAU- Biofungicide}

Seeds were treated with BAU- Biofungicide @ 3\% of seed weight in a $250 \mathrm{ml}$ Erlenmeyer flask and shaken thoroughly for proper coating of the seeds. The treated seeds were kept inside the brown paper bags so that seeds remain in dry condition till for further use.

\section{Experimental design}

The experiments were conducted following Randomized Block Design (RCBD) having three replications. The size of the unit plot was $10 \mathrm{~m}^{2}(5 \mathrm{~m} \times 2 \mathrm{~m})$ and the distance between plots and replications were $1.0 \mathrm{~m}$ and $1.0 \mathrm{~m}$, respectively.

\section{Soil characteristics and nutrient status}

The Soil characteristics and nutrient status of the two experimental stations (JAES, Manikgonj and KRS, Kishoregonj) are shown in (Table 1).

Table I Soil characteristics and nutrient status of the two experimental locations in 2012

\begin{tabular}{|c|c|c|c|c|c|c|c|c|}
\hline \multirow[b]{2}{*}{ Experimental location } & \multirow[b]{2}{*}{ AEZ } & \multicolumn{3}{|c|}{ Soil characteristics } & \multicolumn{4}{|c|}{ Nutrient status } \\
\hline & & land type & Soil Type & $\mathbf{P h}$ & $\begin{array}{l}\% \\
\text { OM }\end{array}$ & $\% \mathbf{N}$ & $\mathbf{P}(\mathbf{P p m})$ & $\mathrm{K}(\mathrm{Meq} / \mathrm{I00})$ \\
\hline $\begin{array}{l}\text { Jute Agriculture Experimental Station } \\
\text { (JAES), Manikgonj, BJRI }\end{array}$ & $\begin{array}{l}\text { Active Brahmaputra and Jamuna } \\
\text { Flood Plain (AEZ-7) }\end{array}$ & $\begin{array}{l}\text { Medium } \\
\text { land }\end{array}$ & $\begin{array}{l}\text { Sandy and } \\
\text { Silty }\end{array}$ & 6.69 & 1.79 & 0.35 & 14.38 & 0.138 \\
\hline $\begin{array}{l}\text { Kishoregonj Regional Station (KRS), } \\
\text { BJRI }\end{array}$ & $\begin{array}{l}\text { Old Brahmaputra Flood Plain } \\
\text { (AEZ-9) }\end{array}$ & $\begin{array}{l}\text { Medium } \\
\text { land }\end{array}$ & Loam & 6.11 & $\mathrm{I} .24$ & 0.39 & 14.98 & 0.15 \\
\hline
\end{tabular}

\section{Application of fertilizers}

\section{Sowing of seeds}

During final land preparation Urea $60 \mathrm{~kg}$, Triple Super Phosphate $50 \mathrm{~kg}$ and Muriate of Potash $25 \mathrm{~kg}$ per hectare were applied. ${ }^{18}$ After 15-20 days of seed germination first top dressing with the urea @ $60 \mathrm{~kg}$ and again another 15 days later of first top dressing, the 2nd top dressing was given with $60 \mathrm{~kg}$ per hectare. Top dressing of urea was done very carefully so that it will not come in contact with the plant parts. To meet sulphur and zinc deficiency, gypsum and zinc oxide @ $45 \mathrm{~kg}$ and $5 \mathrm{~kg}$ per hectare were applied. ${ }^{18}$

Seeds were sown in line on 20 April, 2012 in Kishoregonj Regional Station (KRS), Kishoregonj and $2^{\text {nd }}$ May, 2012 in Jute Agriculture Experimental Station (JAES), Manikgonj. Row to row and plant to plant distance were maintained as $1 \mathrm{M}$ and $1 \mathrm{M}$, respectively. The seed rate for O-9897 was $4 \mathrm{~kg}$ per hectare.

\section{Data collection}

Data on different parameters were collected as shown below

Citation: Haque SMA. Interaction effect among the disease managements, seed treatments and locations in 0-9897 variety on disease incidence, seed yield, stick yield and fiber yield following line sowing method in the field. J Microbiol Exp. 2017;4(2): I I-12. DOI: I0.15406/jmen.2017.04.00 I09 
i. Incidence of diseases $(\%)$

ii. Fibre yield per plant (gm)

iii. Fibre yield per hectare (ton)

iv. Stick yield per plant (gm)

v. Stick yield per hectare (ton)

vi. Average number of branch per plant

vii. Average number of fruits per plant

viii. Seed yield per plant (gm)

ix. Seed yield per hectare $(\mathrm{kg})$

Some plots were kept un-harvested for seed production

\section{Statistical analysis}

Data were analyzed statistically and treatments effects were compared by Duncan's Multiple Range Test (DMRT). Relation between seed borne fungal pathogens and germination was observed with regression equations. Relationships between disease severity and seed, fibre and stick yield were also observed by linear regression lines and equations. ${ }^{19}$

\section{Results}

Interaction effect among the disease managements and seed treatments on disease incidence, seed yield, sticks yield and fibre yield following line sowing method in the field (rewrite sentence)

Interaction effect of different types of disease managements and seed treatments differed significantly for disease incidence, fibre yield, stick yield, number of branch, number of capsule and seed yield (Tables 2,3). In case of JAES, after spraying, interaction effect of BAU- Biofungicide sprayed plants and BAUBiofungicide treated seed resulted lower seed borne infection $(2.53 \%)$ and highest disease incidence $(5.08 \%)$ was encountered in interaction of control and control treatment. Interaction effect between different types of disease managements and seed managements on fibre yield/ha was found significant. But there was no significant differences among $\mathrm{D}_{1} \mathrm{XT}_{1}$ (5.03 ton), $\mathrm{D}_{1} \mathrm{XT}_{2}$ (4.51 ton), $\mathrm{D}_{1} \mathrm{XT}_{3}$ (4.11 ton), $\mathrm{D}_{2} \mathrm{XT}_{1}$ (4.51 ton), $\mathrm{D}_{2} \mathrm{XT}_{2}$ (3.98 ton) and $\mathrm{D}_{3} \mathrm{XT}_{1}$ (4.11 ton). Again there was no significant variation among $\mathrm{D}_{2} \mathrm{XT}_{3}$ (3.59 ton), $\mathrm{D}_{3} \mathrm{XT}_{2}$ (3.59 ton and $\mathrm{D}_{3} \mathrm{XT}_{3}$ (3.19 ton). The highest result was found in $\mathrm{D}_{1} \mathrm{XT}_{1}$ (5.03 ton) followed by $\mathrm{D}_{1} \mathrm{XT}_{2}$ and $\mathrm{D}_{2} \mathrm{XT}_{1}$ (4.51 ton). The lowest result was found in $\mathrm{D}_{3} \mathrm{XT}_{3}$ (3.19 ton) proceeded by $\mathrm{D}_{2} \mathrm{XT}_{3}$ and $\mathrm{D}_{3} \mathrm{XT}_{2}$ (3.59 ton). Interaction effect between locations and different types of disease managements on stick yield/ha were found significant. But there was no significant differences among $\mathrm{D}_{2} \mathrm{XT}_{3}$ (6.58 ton), $\mathrm{D}_{3} \mathrm{XT}_{2}\left(6.81\right.$ ton) and $\mathrm{D}_{3} \mathrm{XT}_{3}(6.14$ ton). Again there was no significant variation among $\mathrm{D}_{2} \mathrm{XT}_{1}$ (8.69 ton) and $\mathrm{D}_{3} \mathrm{XT}_{1}$ (8.25 ton). The highest result was found in $\mathrm{D}_{1} \mathrm{XT}_{1}(10.01$ ton) followed by $\mathrm{D}_{2} \mathrm{XT}_{1}(8.69$ ton). The lowest result was found in $\mathrm{D}_{3} \mathrm{XT}_{3}\left(6.14\right.$ ton) proceeded by $\mathrm{D}_{2} \mathrm{XT}_{3}(6.58$ ton). Interaction effect between locations and different types of disease managements on seed yield/ha was found significant. But there was no significant differences among $\mathrm{D}_{1} \mathrm{XT} \mathrm{T}_{1}(369.02 \mathrm{~kg}), \mathrm{D} 1 \mathrm{XT} 2(359.03 \mathrm{~kg}), \mathrm{D}_{1} \mathrm{XT} \mathrm{T}_{3}(362.79 \mathrm{~kg})$, $\mathrm{D}_{2} \mathrm{XT}_{1}(358.87 \mathrm{~kg}), \mathrm{D}_{2} \mathrm{XT}(348.88 \mathrm{~kg}), \mathrm{D}_{2} \mathrm{XT}_{3}(352.64 \mathrm{~kg}), \mathrm{D}_{3} \mathrm{XT}_{1}$ $(349.98 \mathrm{~kg})$ and $\mathrm{D}_{3} \mathrm{XT}_{2}(343.75 \mathrm{~kg})$. The highest result was found in $\mathrm{D}_{1} \mathrm{XT}_{1}(369.02 \mathrm{~kg})$ followed by D1 XT3 $(362.79 \mathrm{~kg})$. The lowest result was found in $\mathrm{D}_{3} \mathrm{XT}_{3}(339.99 \mathrm{~kg})$ proceeded by $\mathrm{D}_{3} \mathrm{XT}_{2}(343.75 \mathrm{~kg})$ (Table 2).

Table 2 Disease incidence, seed yield, stick yield and fibre yield following line sowing at JAES, BJRI

\begin{tabular}{|c|c|c|c|c|c|c|c|c|c|c|}
\hline $\begin{array}{l}\text { Interaction among } \\
\text { Seed treatments } \\
\text { and disease } \\
\text { managements }\end{array}$ & \multicolumn{2}{|c|}{$\begin{array}{l}\text { Percent disease } \\
\text { incidence }\end{array}$} & $\begin{array}{l}\text { Fibre } \\
\text {-Yield (Gm } \\
\text { /Plant) }\end{array}$ & $\begin{array}{l}\text { Stick Yield } \\
\text { (Gm/Plant) }\end{array}$ & $\begin{array}{l}\text { Fibre Yield } \\
\text { (T/Ha) }\end{array}$ & $\begin{array}{l}\text { Stick Yield } \\
\text { (T/Ha) }\end{array}$ & $\begin{array}{l}\text { No. of branch/ } \\
\text { Plant }\end{array}$ & $\begin{array}{l}\text { No. of Pod/ } \\
\text { Plant }\end{array}$ & $\begin{array}{l}\text { Seed yield } \\
\text { (Gm /Plant) }\end{array}$ & $\begin{array}{l}\text { Seed yield } \\
(\mathrm{Kg} / \mathrm{Ha})\end{array}$ \\
\hline $\mathrm{D}_{1} \times \mathrm{T}_{1}$ & $5.91 \mathrm{e}$ & $2.53 \mathrm{f}$ & $14.67 \mathrm{a}$ & $25.36 \mathrm{a}$ & $5.03 \mathrm{a}$ & $10.01 \mathrm{a}$ & $4.59 \mathrm{a}$ & $26.98 \mathrm{a}$ & 5.18 & $369.02 \mathrm{a}$ \\
\hline $\mathrm{D}_{1} \mathrm{XT}_{2}$ & 6.96 def & $2.7 \mathrm{I}$ ef & I3.95 abc & $24.38 \mathrm{ab}$ & $4.5 \mathrm{I} \mathrm{ab}$ & 8.57 bc & $4.42 \mathrm{ab}$ & $24.32 b$ & 5.14 & $359.03 \mathrm{ab}$ \\
\hline $\mathrm{D}_{1} \mathrm{XT}_{3}$ & $11.01 \mathrm{~b}$ & $3.13 \mathrm{def}$ & I3.39 bcde & 23.59 bc & 4.II ab & $7.90 \mathrm{~cd}$ & $4.25 \mathrm{abcd}$ & $23.32 \mathrm{~b}$ & 4.98 & $362.79 \mathrm{ab}$ \\
\hline $\mathrm{D}_{2} \times \mathrm{T}_{1}$ & 6.84 ef & $3.17 \mathrm{cdef}$ & $14.05 \mathrm{ab}$ & $24.28 \mathrm{ab}$ & $4.5 \mathrm{I} a b$ & $8.69 \mathrm{~b}$ & $4.33 \mathrm{abc}$ & $24.54 b$ & 5.02 & $358.87 \mathrm{ab}$ \\
\hline $\mathrm{D}_{2} \mathrm{XT}_{2}$ & $8.02 \mathrm{~d}$ & $3.60 \mathrm{cde}$ & I3.32 bcde & $23.30 \mathrm{bc}$ & $3.98 \mathrm{ab}$ & $7.25 \mathrm{de}$ & $4.16 \mathrm{bcd}$ & $21.88 \mathrm{c}$ & 4.98 & $348.88 \mathrm{ab}$ \\
\hline $\mathrm{D}_{2} \mathrm{XT}_{3}$ & $12.30 \mathrm{a}$ & $4.08 \mathrm{bc}$ & $12.77 \mathrm{de}$ & $22.51 \mathrm{c}$ & $3.59 \mathrm{~b}$ & 6.58 ef & $3.99 \mathrm{~cd}$ & $20.88 \mathrm{~cd}$ & 4.82 & $352.64 \mathrm{ab}$ \\
\hline $\mathrm{D}_{3} \times \mathrm{T}_{1}$ & 7.69 de & $4.04 \mathrm{bcd}$ & I3.67 bcd & $24.08 \mathrm{ab}$ & 4.II ab & $8.25 \mathrm{bc}$ & $4.21 \mathrm{bcd}$ & $23.67 \mathrm{~b}$ & 4.86 & $349.98 \mathrm{ab}$ \\
\hline $\mathrm{D}_{3} \mathrm{XT}_{2}$ & $9.23 \mathrm{c}$ & $4.67 \mathrm{ab}$ & $12.95 \mathrm{cde}$ & $23.10 \mathrm{bc}$ & $3.59 \mathrm{~b}$ & 6.81 ef & $4.04 \mathrm{bcd}$ & $21.00 \mathrm{~cd}$ & 4.82 & $343.75 \mathrm{ab}$ \\
\hline $\mathrm{D}_{3} \mathrm{XT}_{3}$ & $13.27 \mathrm{a}$ & $5.08 \mathrm{a}$ & $12.39 \mathrm{e}$ & $22.31 \mathrm{c}$ & $3.19 b$ & $6.14 \mathrm{f}$ & $3.87 d$ & $20.00 \mathrm{~d}$ & 4.66 & $339.99 \mathrm{~b}$ \\
\hline Level of significance & 0.05 & 0.05 & 0.05 & 0.05 & 0.05 & 0.05 & 0.05 & 0.05 & NS & 0.05 \\
\hline
\end{tabular}

$\mathrm{D}_{1}$, Spraying of BAU- Biofungicide (3\%); $\mathrm{D}_{2}$, Spraying of Dithane M-45 (2\%); $\mathrm{D}_{3}$, Control (No spray); $\mathrm{T}_{1}$, Seed treated with BAU- Biofungicide (3\%); $\mathrm{T}_{2}$, Seed treated with Provax-200 (0.4\%); $\mathrm{T}_{3}$, Control (No spray) Data in column having common letter(s) do not differ significantly at $5 \%$ level of significance.

NS, Not Significant

In case of KRS, after spraying, interaction effect of BAUBiofungicide sprayed plants and BAU- Biofungicide treated seed resulted lower seed borne infection $(1.36 \%)$ and highest disease incidence $(3.46 \%)$ was encountered in interaction of control and control treatment (Table 3). Interactions effect between different types of disease managements and seed managements on fibre yield/ha were found no significant. The highest result was found in $\mathrm{D}_{1} \mathrm{XT}_{1}(3.97$ ton) followed by $\mathrm{D}_{1} \mathrm{XT}_{2}$ and $\mathrm{D}_{2} \mathrm{XT}_{1}$ (3.93ton). The lowest result was found in $\mathrm{D}_{3} \mathrm{XT}_{3}$ (2.85 ton) proceeded by $\mathrm{D}_{2} \mathrm{XT}_{3}$ and $\mathrm{D}_{3} \mathrm{XT}_{2}$ (3.37 ton). Interaction effect between locations and different types of disease managements on stick yield/ha were found significant. But there was no significant differences among $\mathrm{D}_{1} \mathrm{XT}_{1}$ (8.82 ton), $\mathrm{D}_{1} \mathrm{XT}_{2}$ (8.21 ton) and $\mathrm{D}_{2} \mathrm{XT}_{1}$ (8.15 ton). Again there was no significant variation among $\mathrm{D}_{1} \mathrm{XT}_{3}$ (7.18 ton), $\mathrm{D}_{3} \mathrm{XT}_{1}$ (7.31 ton) and $\mathrm{D}_{3} \mathrm{XT}_{2}$ ( 6.70 ton). The highest result was found in $\mathrm{D}_{1} \mathrm{XT}_{1}$ (8.82ton) followed by $\mathrm{D}_{1} \mathrm{XT}_{2}(8.21$ ton). The lowest result was found in $\mathrm{D}_{3} \mathrm{XT}_{3}$ (5.67 ton) proceeded by $\mathrm{D}_{2} \mathrm{XT}_{3}$ (6.51ton). Interactions effect between locations and different types of disease managements on seed yield/ha were found significant. But there was no significant differences among $\mathrm{D}_{1} \mathrm{XT} \mathrm{T}_{1}(373.54 \mathrm{~kg})$, $\mathrm{D}_{1} \mathrm{XT}_{2}(363.89 \mathrm{~kg}), \mathrm{D}_{1} \mathrm{XT}_{3}(373.68 \mathrm{~kg}), \mathrm{D}_{2} \mathrm{XT}_{1}(362.50 \mathrm{~kg})$, $\mathrm{D}_{2} \mathrm{XT}_{2}(353.85 \mathrm{~kg}), \mathrm{D}_{2} \mathrm{XT}_{3}(362.65 \mathrm{~kg}), \mathrm{D}_{3} \mathrm{XT}_{1}(355.03 \mathrm{~kg})$ and $\mathrm{D}_{3} \mathrm{XT}_{2}(355.17 \mathrm{~kg})$. The highest result was found in $\mathrm{D}_{1} \mathrm{XT}_{3}(373.68$ 
$\mathrm{kg})$ followed by $\mathrm{D}_{1} \mathrm{XT} \mathrm{T}_{1}(373.54 \mathrm{~kg})$. The lowest result was found in $\mathrm{D}_{3} \mathrm{XT}_{3}(345.38 \mathrm{~kg})$ preceeded by $\mathrm{D}_{3} \mathrm{XT}_{1}(355.03 \mathrm{~kg})$ (Table 3$)$.

Interaction effect among the locations, disease managements and seed treatments on disease incidence, seed yield, stick yield and fibre yield following line sowing method in the field

Interaction effect of locations, different types of disease managements and seed treatments differed significantly for disease incidence, fibre yield, stick yield, number of branch, number of capsule and seed yield (Table 4). After spraying, interaction effect of KRS, BAU- Biofungicide sprayed plants and BAU- Biofungicide treated seed resulted lower seed borne infection (1.36\%) and highest disease incidence $(5.08 \%)$ was encountered in interaction of JAES, control and control treatment. Interactions effect between locations and different types of disease managements and seed managements on fibre yield/ha were found significant. But there was no significant differences among $\mathrm{L}_{1} \mathrm{XD}_{1} \mathrm{XT}_{1}$ (5.03 ton), $\mathrm{L}_{1} \mathrm{XD}_{1} \mathrm{XT}_{2}$ (4.51ton), $\mathrm{L}_{1} \mathrm{XD}_{1} \mathrm{XT}_{3}$ (4.11 ton), $\mathrm{L}_{1} \mathrm{XD}_{2} \mathrm{XT}_{1}$ (4.51 ton), $\mathrm{L}_{1} \mathrm{XD}_{2} \mathrm{XT}_{2}$ (3.98 ton), $\mathrm{L}_{1} \mathrm{XD}_{3} \mathrm{XT}_{1}$ (4.11 ton), $\mathrm{L}_{2} \mathrm{XD}_{1} \mathrm{XT}_{1}$ (3.97 ton), $\mathrm{L}_{2} \mathrm{XD}_{1} \mathrm{XT}_{2}$ (3.93 ton), $\mathrm{L}_{2} \mathrm{XD}_{2} \mathrm{XT}_{1}$ (3.93 ton) and $\mathrm{L}_{2} \mathrm{XD}_{2} \mathrm{XT}_{2}$ (3.37 ton). Again there was no significant variation among $\mathrm{L}_{1} \mathrm{XD}_{2} \mathrm{XT}_{3}$ (3.59 ton), $\mathrm{L}_{1} \mathrm{XD}_{3} \mathrm{XT}_{2}$ (3.59 ton), $\mathrm{L}_{1} \mathrm{XD}_{3} \mathrm{XT}_{3}$ (3.19 ton), $\mathrm{L}_{2} \mathrm{XD}_{1} \mathrm{XT}_{1}$ (3.41 ton), $\mathrm{L}_{2} \mathrm{XD}_{2} \mathrm{XT}_{3}$ (3.37 ton), $\mathrm{L}_{2} \mathrm{XD}_{3} \mathrm{XT}_{1}$ (3.41 ton) and $\mathrm{L}_{2} \mathrm{XD}_{3} \mathrm{XT}_{2}$ (3.37 ton). The highest result was found in $\mathrm{L}_{1} \mathrm{XD}_{1} \mathrm{XT}_{1}$

Table 3 Interaction effects among the disease managements and seed treatments in 0-9897 variety on disease incidence, seed yield, stick yield and fibre yield following line sowing at KRS, BJRI

\begin{tabular}{|c|c|c|c|c|c|c|c|c|c|c|}
\hline \multirow{2}{*}{$\begin{array}{l}\text { Interaction among } \\
\text { Seed treatments } \\
\text { and disease } \\
\text { managements }\end{array}$} & \multicolumn{2}{|c|}{$\begin{array}{l}\text { Percent Disease } \\
\text { Incidence }\end{array}$} & \multirow{2}{*}{$\begin{array}{l}\text { Fibre } \\
\text {-yield (Gm } \\
\text { /Plant) }\end{array}$} & \multirow{2}{*}{$\begin{array}{l}\text { Stick Yield } \\
\text { (Gm/Plant) }\end{array}$} & \multirow{2}{*}{$\begin{array}{l}\text { Fibre } \\
\text { Yield (TI } \\
\text { Ha) }\end{array}$} & \multirow{2}{*}{$\begin{array}{l}\text { Stick } \\
\text { Yield (T/ } \\
\text { Ha) }\end{array}$} & \multirow{2}{*}{$\begin{array}{l}\text { No. of Branch/ } \\
\text { Plant }\end{array}$} & \multirow{2}{*}{$\begin{array}{l}\text { No. of Pod } / \\
\text { Plant }\end{array}$} & \multirow{2}{*}{$\begin{array}{l}\text { Seed yield } \\
\text { (Gm / } \\
\text { Plant) }\end{array}$} & \multirow{2}{*}{$\begin{array}{l}\text { Seed yield } \\
(\mathrm{Kg} / \mathrm{Ha})\end{array}$} \\
\hline & Before & After & & & & & & & & \\
\hline $\mathrm{D}_{1} \times \mathrm{T}_{1}$ & $3.89 \mathrm{f}$ & $1.36 \mathrm{~b}$ & $1 \mathrm{I} .15 \mathrm{a}$ & $24.28 a$ & 3.97 & $8.82 a$ & $4.09 a$ & $25.62 \mathrm{a}$ & 5.42 & $373.54 a$ \\
\hline $\mathrm{D}_{1} \times \mathrm{T}_{2}$ & $5.01 \mathrm{e}$ & $1.69 \mathrm{~b}$ & $11.10 \mathrm{a}$ & $24.00 \mathrm{ab}$ & 3.93 & $8.21 \mathrm{lab}$ & $3.99 \mathrm{ab}$ & $25.32 \mathrm{a}$ & 5.31 & $363.89 \mathrm{ab}$ \\
\hline $\mathrm{D}_{1} \mathrm{XT}_{3}$ & $6.16 \mathrm{~d}$ & $2.11 \mathrm{ab}$ & $10.65 \mathrm{ab}$ & $23.40 \mathrm{abc}$ & 3.41 & $7.18 \mathrm{cde}$ & $3.87 a b$ & $22.83 \mathrm{bc}$ & 5.24 & $373.68 \mathrm{a}$ \\
\hline $\mathrm{D}_{2} \times \mathrm{T}_{1}$ & $5.17 \mathrm{e}$ & $2.02 \mathrm{ab}$ & $10.55 \mathrm{ab}$ & $23.33 \mathrm{abc}$ & 3.93 & $8.15 \mathrm{ab}$ & $3.98 \mathrm{ab}$ & $23.47 \mathrm{~b}$ & 5.28 & $362.50 \mathrm{ab}$ \\
\hline $\mathrm{D}_{2} \mathrm{XT}_{2}$ & $6.55 \mathrm{~d}$ & $2.14 \mathrm{ab}$ & $10.50 \mathrm{ab}$ & 23.05 bc & 3.89 & 7.54 bc & $3.88 a b$ & 23.17 bc & 5.17 & $352.85 \mathrm{ab}$ \\
\hline $\mathrm{D}_{2}^{2} \mathrm{XT}_{3}^{2}$ & $7.76 \mathrm{c}$ & $2.60 \mathrm{ab}$ & $10.05 b c$ & $22.46 \mathrm{~cd}$ & 3.37 & $6.51 \mathrm{e}$ & $3.75 a b$ & $20.68 d$ & 5.10 & $362.65 \mathrm{ab}$ \\
\hline $\mathrm{D}_{3} \times \mathrm{T}_{1}$ & $8.23 c$ & $2.69 \mathrm{ab}$ & $9.97 \mathrm{bc}$ & $22.74 \mathrm{~cd}$ & 3.41 & $7.31 \mathrm{~cd}$ & $3.85 \mathrm{ab}$ & 22.43 bc & 5.05 & $355.03 \mathrm{ab}$ \\
\hline $\mathrm{D}_{3} \mathrm{XT}_{2}$ & $9.03 \mathrm{~b}$ & $2.84 \mathrm{ab}$ & $9.93 \mathrm{bc}$ & $22.46 \mathrm{~cd}$ & 3.37 & $6.70 \mathrm{de}$ & $3.75 \mathrm{ab}$ & $22.13 \mathrm{c}$ & 4.94 & $355.17 \mathrm{ab}$ \\
\hline $\mathrm{D}_{3} \mathrm{XT}_{3}$ & $10.29 \mathrm{a}$ & $3.46 \mathrm{a}$ & $9.47 \mathrm{c}$ & $21.86 \mathrm{~d}$ & 2.85 & $5.67 \mathrm{f}$ & $3.62 b$ & $19.64 \mathrm{e}$ & 4.87 & $345.38 b$ \\
\hline Level of significance & 0.05 & 0.05 & 0.05 & 0.05 & NS & 0.05 & 0.05 & 0.05 & NS & 0.05 \\
\hline
\end{tabular}

$\mathrm{D}_{\text {, }}$ Spraying of BAU- Biofungicide (3\%); $\mathrm{D}_{2}$, Spraying of Dithane M-45 (2\%); $\mathrm{D}_{3}$, Control (No spray); $\mathrm{T}_{\mathrm{l}}$, Seed treated with BAU- Biofungicide (3\%); $\mathrm{T}_{2}$, Seed treated with Provax-200 (0.4\%); $T_{3}$, Control (No spray)

Data in column having common letter(s) do not differ significantly at $5 \%$ level of significance.

NS, Not Significant

Table 4 Interaction effect among the location, disease managements and seed treatments in O-9897 variety on disease incidence, seed yield, stick yield and fibre yield following line sowing method in the field

\begin{tabular}{|c|c|c|c|c|c|c|c|c|c|c|}
\hline $\begin{array}{l}\text { Interaction } \\
\text { among locations, } \\
\text { seed treatments } \\
\text { and disease } \\
\text { managements }\end{array}$ & $\begin{array}{l}\text { Percent } \\
\text { inciden } \\
\text { before }\end{array}$ & ease & $\begin{array}{l}\text { Fibre } \\
\text { Yield } \\
\text { (Gm / } \\
\text { Plant) }\end{array}$ & $\begin{array}{l}\text { Stick Yield } \\
\text { (Gm/Plant) }\end{array}$ & $\begin{array}{l}\text { Fibre } \\
\text { Yield } \\
(\mathrm{T} / \mathrm{Ha})\end{array}$ & $\begin{array}{l}\text { Stick } \\
\text { Yield } \\
\text { (T/Ha) }\end{array}$ & $\begin{array}{l}\text { No. of Branch/ } \\
\text { Plant }\end{array}$ & $\begin{array}{l}\text { No. of Pod/ } \\
\text { Plant }\end{array}$ & $\begin{array}{l}\text { Seed Yield } \\
\text { (Gm/Plant) }\end{array}$ & $\begin{array}{l}\text { Seed Yield (Kg/ } \\
\mathrm{Ha})\end{array}$ \\
\hline $\mathrm{L}_{1} \times \mathrm{D}_{1} \times \mathrm{T}_{1}$ & $5.91 \mathrm{hi}$ & $2.53 \mathrm{defg}$ & $14.67 \mathrm{a}$ & $25.36 \mathrm{a}$ & $5.03 \mathrm{a}$ & $10.01 \mathrm{a}$ & $4.59 \mathrm{a}$ & $26.98 \mathrm{a}$ & 5.18 & $369.02 \mathrm{ab}$ \\
\hline $\mathrm{L}_{1} \times \mathrm{D}_{1} \times \mathrm{T}_{2}$ & $6.96 \mathrm{fg}$ & $2.71 \mathrm{def}$ & $13.95 \mathrm{ab}$ & $24.38 \mathrm{abc}$ & $4.5 \mathrm{I} \mathrm{ab}$ & 8.57 bc & $4.42 \mathrm{ab}$ & $24.32 \mathrm{bcd}$ & 5.14 & $359.03 \mathrm{abc}$ \\
\hline $\mathrm{L}_{1} \times \mathrm{D}_{1} \times \mathrm{T}_{3}^{2}$ & $11.01 \mathrm{c}$ & $3.13 \mathrm{cde}$ & $13.39 \mathrm{abc}$ & $23.59 \mathrm{bcde}$ & 4.II abc & $7.90 \mathrm{de}$ & $4.25 \mathrm{abcd}$ & 23.32 cdefg & 4.98 & $362.79 \mathrm{abc}$ \\
\hline$L_{1} \times D_{2} \times T_{1}$ & $6.84 \mathrm{~g}$ & $3.17 \mathrm{cde}$ & $14.05 \mathrm{ab}$ & $24.28 \mathrm{abcd}$ & $4.5 \mathrm{I} \mathrm{ab}$ & 8.69 bc & $4.33 \mathrm{abc}$ & 24.54 bc & 5.02 & $358.87 \mathrm{abc}$ \\
\hline $\mathrm{L}_{1} \times \mathrm{D}_{2} \times \mathrm{T}_{2}$ & $8.02 \mathrm{e}$ & $3.60 \mathrm{bcd}$ & I3.32 bc & $23.30 \mathrm{cdef}$ & $3.98 \mathrm{abc}$ & $7.25 \mathrm{fg}$ & 4. $16 \mathrm{bcde}$ & $21.88 \mathrm{ghi}$ & 4.98 & 348.88 bc \\
\hline $\mathrm{L}_{1} \times \mathrm{D}_{2}^{2} \times \mathrm{T}_{3}^{2}$ & $12.30 \mathrm{~b}$ & $4.08 \mathrm{abc}$ & $12.77 \mathrm{bc}$ & 22.51 efg & $3.59 \mathrm{bc}$ & $6.58 \mathrm{hi}$ & $3.99 \mathrm{cdef}$ & $20.88 \mathrm{ij}$ & 4.82 & $352.64 \mathrm{abc}$ \\
\hline $\mathrm{L}_{1}^{1} \times \mathrm{D}_{3}^{2} \times \mathrm{T}_{1}^{3}$ & 7.69 ef & $4.04 \mathrm{abc}$ & I $3.67 \mathrm{abc}$ & $24.08 \mathrm{bcd}$ & $4.11 \mathrm{abc}$ & $8.25 \mathrm{bcd}$ & $4.21 \mathrm{abcd}$ & $23.67 \mathrm{cde}$ & 4.86 & $349.98 \mathrm{abc}$ \\
\hline$L_{1} \times D_{3} \times T_{2}$ & $9.23 \mathrm{~d}$ & $4.67 \mathrm{ab}$ & $12.95 \mathrm{bc}$ & $23.10 \mathrm{def}$ & $3.59 \mathrm{bc}$ & $6.81 \mathrm{gh}$ & 4.04 bcdef & $21.00 \mathrm{hij}$ & 4.82 & $339.99 \mathrm{c}$ \\
\hline $\mathrm{L}_{1} \times \mathrm{D}_{3}^{3} \times \mathrm{T}_{3}^{2}$ & $13.27 \mathrm{a}$ & $5.08 \mathrm{a}$ & $12.39 \mathrm{c}$ & $22.31 \mathrm{fg}$ & $3.19 b c$ & $6.14 \mathrm{ij}$ & 3.87 def & $20.00 j$ & 4.66 & $343.75 c$ \\
\hline$L_{2} \times D_{1}^{3} \times T_{1}^{3}$ & $3.89 \mathrm{k}$ & $1.36 \mathrm{~g}$ & $11.15 \mathrm{~d}$ & $24.28 \mathrm{abcd}$ & $3.97 \mathrm{abc}$ & $8.82 b$ & 4.09 bcde & $25.62 b$ & 5.42 & $373.54 \mathrm{a}$ \\
\hline $\mathrm{L}_{2} \times \mathrm{D}_{1} \times \mathrm{T}_{2}$ & $5.01 \mathrm{j}$ & $1.69 \mathrm{fg}$ & $11.10 \mathrm{~d}$ & $24.00 \mathrm{bcd}$ & $3.93 \mathrm{abc}$ & $8.21 \mathrm{bcd}$ & $3.99 \mathrm{cdef}$ & $25.32 \mathrm{~b}$ & 5.31 & $363.89 \mathrm{abc}$ \\
\hline $\mathrm{L}_{2} \times \mathrm{D}_{1} \times \mathrm{T}_{3}$ & $6.16 \mathrm{gh}$ & 2.II efg & $10.65 \mathrm{de}$ & 23.40 cdef & $3.4 \mathrm{I} \mathrm{bc}$ & $7.18 \mathrm{fg}$ & $3.87 \mathrm{def}$ & 22.83 defg & 5.24 & $373.68 \mathrm{a}$ \\
\hline $\mathrm{L}_{2} \times \mathrm{D}_{2} \times \mathrm{T}_{1}$ & $5.17 \mathrm{ij}$ & 2.02 efg & $10.55 \mathrm{de}$ & $23.33 \mathrm{cdef}$ & $3.93 \mathrm{abc}$ & $8.15 \mathrm{~cd}$ & $3.98 \mathrm{cdef}$ & $23.47 \mathrm{cdef}$ & 5.28 & $362.50 \mathrm{abc}$ \\
\hline $\mathrm{L}_{2}^{2} \times \mathrm{D}_{2} \times \mathrm{T}_{2}$ & $6.55 \mathrm{gh}$ & 2.14 efg & $10.50 \mathrm{de}$ & 23.05 defg & $3.89 \mathrm{abc}$ & 7.54 ef & $3.88 \mathrm{def}$ & 23.17 cdefg & 5.17 & $352.85 \mathrm{abc}$ \\
\hline $\mathrm{L}_{2} \times \mathrm{D}_{2} \times \mathrm{T}_{3}$ & 7.76 ef & $2.60 \mathrm{def}$ & $10.05 \mathrm{de}$ & $22.46 \mathrm{efg}$ & $3.37 b c$ & $6.5 \mathrm{I} \mathrm{hi}$ & 3.75 ef & $20.68 \mathrm{ij}$ & 5.1 & $362.65 \mathrm{abc}$ \\
\hline $\mathrm{L}_{2} \times \mathrm{D}_{3} \times \mathrm{T}_{1}$ & $8.23 \mathrm{e}$ & $2.69 \mathrm{def}$ & $9.97 \mathrm{de}$ & $22.74 \mathrm{ab}$ & $3.4 I \mathrm{bc}$ & $7.31 \mathrm{fg}$ & 3.85 def & 22.43 efgh & 5.05 & $355.03 \mathrm{abc}$ \\
\hline$L_{2}^{2} \times D_{3} \times T_{2}$ & $9.03 \mathrm{~d}$ & 2.84 def & $9.93 \mathrm{de}$ & $22.46 \mathrm{efg}$ & $3.37 b c$ & $6.70 \mathrm{chi}$ & 3.75 ef & $22.13 \mathrm{fghi}$ & 4.94 & $345.38 \mathrm{bc}$ \\
\hline $\mathrm{L}_{2} \times \mathrm{D}_{3} \times \mathrm{T}_{3}$ & $10.29 \mathrm{c}$ & $3.46 \mathrm{~cd}$ & $9.47 \mathrm{e}$ & $21.86 \mathrm{~g}$ & $2.85 \mathrm{c}$ & $5.67 \mathrm{j}$ & $3.62 \mathrm{f}$ & $19.64 j$ & 4.87 & $355.17 \mathrm{abc}$ \\
\hline Level of significance & 0.05 & 0.05 & 0.05 & 0.05 & 0.05 & 0.05 & 0.05 & 0.05 & NS & 0.05 \\
\hline
\end{tabular}

$L_{\text {, }}$ JAES, Manikgonj, BJRI; $L_{2}$, KRS, Kishoregonj; BJRI, D, Spraying of BAU- Biofungicide (3\%); $D_{2}$, Spraying of Dithane M-45 (2\%); $D_{3}$, Control (No spray); $T_{1}$, Seed treated with BAU- Biofungicide (3\%); $\mathrm{T}_{2}$, Seed treated with Provax-200 (0.4\%); $\mathrm{T}_{3}$, Control (No spray)

Data in column having common letter(s) do not differ significantly at $5 \%$ level of significance.

NS, Not Significant

Citation: Haque SMA. Interaction effect among the disease managements, seed treatments and locations in 0-9897 variety on disease incidence, seed yield, stick yield and fiber yield following line sowing method in the field.J Microbiol Exp. 2017;4(2):I I-I2. DOI: I0.15406/jmen.2017.04.00 I09 


\section{Discussion}

In field experiment, two disease practices viz. spraying of BAUBiofungicide and Dithane M- 45 and moisture content ( $9.5 \%$ moisture) were used for this study. Field experiments were conducted at two different locations- Jute Agriculture Experimental Station (JAES), Manikgonj and Kishoregonj Regional Station (KRS), Kishoregonj of Bangladesh Jute Research Institute. Under field condition, effects of BAU- Biofungicide and Dithane M-45 on disease incidence and production of quality healthy seeds were studied. Interaction between disease managements and seed treatments, in case of JAES, after spraying, lower seed borne infection $(2.53 \%)$ was observed in interaction effect of BAU- Biofungicide spraying plants and BAUBiofungicide treated seed and highest disease incidence $(5.08 \%)$ was encountered in interaction effect among control condition and control treatment. In case of KRS, after spraying, interaction effect of BAU- Biofungicide spraying plants and BAU- Biofungicide treated seed resulted lower seed borne infection (1.39\%) and highest disease incidence $(3.46 \%)$ was encountered in interaction of control and control treatment. Interaction between disease managements, seed treatments and locations, after spraying, interaction effect of KRS, BAU- Biofungicide spraying plants and BAU- Biofungicide treated seed resulted lower seed borne infection $(1.36 \%)$ and highest disease incidence $(5.08 \%)$ was encountered in interaction effect of JAES, control condition and control treatment.. BAU- Biofungicide and Dithane M-45 have been recorded as superior means for controlling seed borne fungi as well as field fungi with higher seed yield and better improvement of seed quality as reported by. ${ }^{18,20-27}$ The present findings revealed that disease incidence was comparatively lower in case of using BAU- Biofungicide and Dithane M-45 spraying. Moreover, management of disease by BAU- Biofungicide increased yield with the decrease of total seed borne fungal pathogens. Ahmed reported that among the causal agents of jute diseases, fungal pathogens are the main group of organisms responsible for the loss of fibre yield. Ahmed $^{12}$ also reported that three important diseases as stem rot (Macrophomina phaseolina), anthracnose (Colletrotichum corchori) and leaf mosaic (virus) disease that deteriorated jute seed. ${ }^{21,25,26,28,29}$ similarly reported that BAU- Biofungicide (3\%) was found to control the seed borne pathogens and also increased the yield (seed and fibre). The low yield in control was related to high prevalence of seed borne fungal infections. Similar result was also reported by Biswas $\mathrm{AC}^{14}$ Interaction between disease managements and seed treatments, in case of JAES, fibre yield/ha (5.03 ton), stick yield/ha (10.01 ton) and seed yield/ ha $(369.02 \mathrm{~kg})$ were recorded in interaction effect of BAUBiofungicide spraying plants and BAU-Biofungicide treated seed. In case of KRS, fibre yield/ha (3.97 ton), stick yield/ha (8.82 ton) and seed yield/ ha $(373.54 \mathrm{~kg})$ were recorded in interaction effect of BAU- Biofungicide spraying plants and BAU- Biofungicide treated seed. Interaction between disease managements, seed treatments and locations, the highest fibre yield/ha (5.03 ton) and stick yield/ha (10.01 ton) were recorded in interaction effect of JAES, BAU- Biofungicide spraying plants and BAU- Biofungicide treated seed. Highest seed yield $/ \mathrm{ha}(373.54 \mathrm{~kg})$ were recorded in interaction effect of KRS, BAUBiofungicide spraying plants and BAU- Biofungicide treated seed.

\section{Conclusion}

Therefore, the following conclusion may be drawn for quality seed and fibre production from the findings of this study:

i. BAU- Biofungicide ( $2 \%$ in water) can successfully used as spraying agent to avoid Dithane M- 45 or other chemical fungicides for the production of quality healthy jute seeds with higher seed and fibre yield. ii. Fibre and seed yield were found to decrease with the increase of seed borne infection of fungal pathogens.

iii. Seed germination and disease incidence varied significantly with respect to disease management, seed treatment and location.

So, the following recommendation may be drawn for quality seed and fibre production from the findings of this study:

i. Quality of jute seeds can be maintained by spraying of BAUBiofungicide and seed treated of BAU- Biofungicide enhance the quality and yield of the jute seed and fibre in the field.

\section{Conflicts of Interest}

The authors declare no conflict of interest.

\section{Acknowledgments}

None.

\section{Funding}

None.

\section{References}

1. Islam MM. About jute seed research. RS printing press, Dhaka, Bangladesh. 2007;1216. 1

2. IJSG. Internal Jute Study Group. Dhaka, Bangladesh. 2012-13.

3. BBS. Statistical Year Book of Bangladesh. Bangladesh Bureau of Statistics, Planning Ministry, Dhaka, Bangladesh. 2011.

4. Khandakar AL. Jute seed at farm level. Food and agriculture Organization of the United nation, Bangladesh. 1987;p. 1-77.

5. Hossain MA, Talukder FAH, Islam M, et al. Studies on C. olitorius pipeline varieties. Ann Rep Bangladesh Jute Res Inst Dhaka. 1994;132-137.

6. Fakir GA, Islam MR. Survey on the health status of jute and rice seeds of farmers of Sadar Upazilla, Mymensingh. BAURES progress. 1990;4: $42-47$.

7. Fakir GA. An annotated list of seed borne diseases in Bangladesh. Seed Pathology Laboratory Dept Plant Path BAU Mymensingh. 2001;p. 7-8.

8. Ikata S, Yoshida M. A new anthracnose of jute plant. Annuals of Phytopath. 1940;10(2-3):141-149.

9. Ahmed QA. Problems in jute plant pathology. Plant Pathology Journal. 1966;184-186.

10. Fakir GA, Sarder MA, Gaffar A, et al. An annotated list of important disorders of important crop plants of Bangladesh. Pl. Quarantine Rev Prog Sponsored by the Ministry of Agriculture in coordination with CIDA and DANIDA. 1991; p.107.

11. Fazli SFI, Ahmed QA. Fungus organisms associated with jute seeds and their effect on germinating seeds and seedlings. Agric Pakistan. 1960;11: 298-306.

12. Ahmed QA. Diseases of jute in East Pakistan. International Journal of phytopathology. 1968;7:147-151.

13. Ahmed N, Islam N. Correlation between jute seed infections recorded in the laboratory with the incidence diseases in the field. Ann Rep BJRI. $1980 ; 129-130$.

14. Biswas AC, Taher MA, Asaduzzaman M, et al. Loss of yield and quality of jute fibre due to prevalence of stem rot. J Plant Pathol. 1985;1(1):6162 .

15. Ahmed N, Sultana K. Survey on the production and quality of jute seeds at Farm level. Ann Rep BJRI. 1985;296-323. 
16. Fakir GA. Seed health test in seed quality control and seed certification Seed Path Lab. 1989;4:11.

17. Hossain I. b BAU-Bio fungicide: Unique eco - friendly means and new dimension of plant disease control in Bangladesh. Lima Printing Press, Mymensingh, Bangladesh. 2011;p .07.

18. Hossain I, Sarkar SR. Nursery diseases of mango and their management. Proce of Bangladesh Agricultural University Research Progress. 2008;10(2):37-38.

19. Gomez A K, Gomez AA. Statistical Procedures for Agric. Res Second Edn Intl Rice Res Inst Manila. 1984;p. 680.

20. Agarwal VK, Singh OV. Seed borne fungi of jute and their control. Indian Phytopathol. 1974;27(4): 651-652.

21. Hossain I. Troymashik Krishi Projuktii Barta. BAU Res. (2011a);3(8):1315.

22. Hossain I, Hossain MM. Status of tikka disease of groundnut in Bangladesh and effect of BAU- Biofungicide on improving seed quality. BAU Res Prog. 2010;20:29-30.

23. Hossain I, Sultana I. Advanced of biocontrol means over chemicals in controlling seed and seedling diseases of maize. Int J Sustain Agril Tech. 2011;7(5):21-27.
24. Islam N, Biswas AC. Screening of seed dressing fungicides. Annual Report BJRI. 1981; p. 218-219.

25. Mostafa G. Effect of BAU- Biofungicide in comparison with Bavistin and Amistar for controlling diseases of soybean variety G2 An MS Thesis submitted to the Department of Plant Pathology, Mymensingh, Bangladesh. 2009

26. Shultana R, Hossain I, Ahmed S, et al. Efficacy of BAU- Biofungicide in controlling leaf spot of wheat (Triticumaestivum). Eco-friendly Agril J. 2009;2(2):392-395.

27. Yeasmin R, Hossain I, Hossain MM. Management of seedling diseases of blackgram, mungbean and lentil using BAU- Biofungicide, biofertilizer and cowdung. Eco-friendly Agril J. 2009;2(11):905-910.

28. Islam MM. Jute seed technology. College gate binding and printing, Dhaka, Bangladesh. 2009;p. 89-97.

29. Islam MM, Rahman M. Hand book on Agricultural technology of jute, kenaf and mesta crops. Bulbul printers, Dhaka, Bangladesh. 2008;p. $27-28$. 